9. Gupta AK, Ghosh B, Mazumdar S, Gupta A. An unusual intraocular foreign body. Acta Ophthalmol (Scand) 1996;74:200-1.

10. Chang WJ, Rapuano CJ. Management of an anterior chamber eyelash. Ophthalmic Surg 1994;25:741-2.

Abraham Solomon

Itai Chowers

Joseph Frucht-Pery

Department of Ophthalmology

Hadassah University Hospital

and

Hebrew University - Hadassah Medical School

Jerusalem

Israel

Abraham Solomon, MD

Department of Ophthalmology

Hadassah University Hospital

PO Box 12000

91120 Jerusalem

Israe

Fax: +97226434434

e-mail: avisol@md2.huji.ac.il

Sir,

\section{Pseudoexfoliation syndrome and spontaneous lens dislocation}

Pseudoexfoliation syndrome (PXS) is characterised by deposits of white, fibrillogranular material in and around the eye. Its association with secondary open angle glaucoma, ${ }_{1}^{1}$ poor pupillary dilatation ${ }^{2}$ and predisposition to zonular dehiscence during cataract surgery ${ }^{3}$ is well known. We report an unusual case of bilateral spontaneous dislocation of lens in a patient with PXS and discuss its implications.

\section{Case report}

An 88-year-old white woman attended our clinic in early 1998 for monitoring of her glaucoma. She complained of sudden deterioration of vision 2 months prior to this visit. There was no history of trauma. In 1981 she had been diagnosed as having open angle glaucoma secondary to PXS. In 1986 she had simultaneous bilateral trabeculectomies due to progressive visual field loss despite maximal topical therapy. The surgery was uncomplicated and post-operatively her intraocular pressures were well controlled without any medication. Her vision gradually deteriorated due to increasing lens opacities. In 1996, with a visual acuity of 6/18 right and $6 / 24$ left, she was offered cataract surgery. The patient decided against it.

At her appointment in January 1998 the uncorrected vision was less than 6/60 in both eyes. Intraocular pressures were normal and functioning blebs with peripheral iridotomies were noted. PXM

(pseudoexfoliative material) was present at the pupillary margins. She was aphakic in her right eye (Fig. 1) and fundus examination revealed the lens to be dislocated inferiorly in the vitreous. The left lens had subluxated inferiorly and the superior equator of the lens with PXM was visible in the mid-pupillary area (Fig. 2). There was no evidence of uveitis and the cup:disc ratio was 0.80 in both eyes. Her visual acuity was improved with an aphakic correction to 6/9 right and 6/24 left. She was given a spherical prescription of +11.75 dioptres right and +6.00 dioptres left. As the patient was pleased with her vision and there were no complications due to the dislocated lenses, surgical intervention was thought to be unnecessary.

\section{Comment}

Recent studies have demonstrated that pseudoexfoliation is a systemic disorder affecting various organs. ${ }^{4}$ There is an association with elastosis ${ }^{5}$ and an increased vascular risk has also been suggested. ${ }^{6}$ However, the main cause for concern for an ophthalmic surgeon is the predisposition of these patients to poor pupillary dilatation and weak lens zonule, as these can contribute to intra-operative complications such as vitreous loss.

The only previous publication on spontaneous lens dislocation in PXS reported two patients with unilateral dislocated lenses. ${ }^{7}$ Both these patients were, like the patient in our report, in their mid- to late eighties. It seems that increasing age and the progressive nature of PXS combine to further weaken the lens zonules resulting in spontaneous lens dislocation. Although our patient had previous bilateral trabeculectomies she was not noted to have damaged her zonules during the procedure. In the long follow-up period there were no signs suggestive of subluxated lenses.

Our case demonstrates that PXS should be considered as a differential diagnosis in older patients with spontaneous lens dislocation. This case further emphasises the extreme care that needs to be taken while performing cataract surgery on elderly patients with PXS. During phacoemulsification, careful grooving technique must be used to minimise physical forces on the lens nucleus and zonules. Cracking technique may put undue strain on lens zonules and therefore a chopping technique is preferred. Patients with PXS also need to be made aware of the possibility of spontaneous lens dislocation and there may be an argument for performing cataract surgery early in this group of patients.

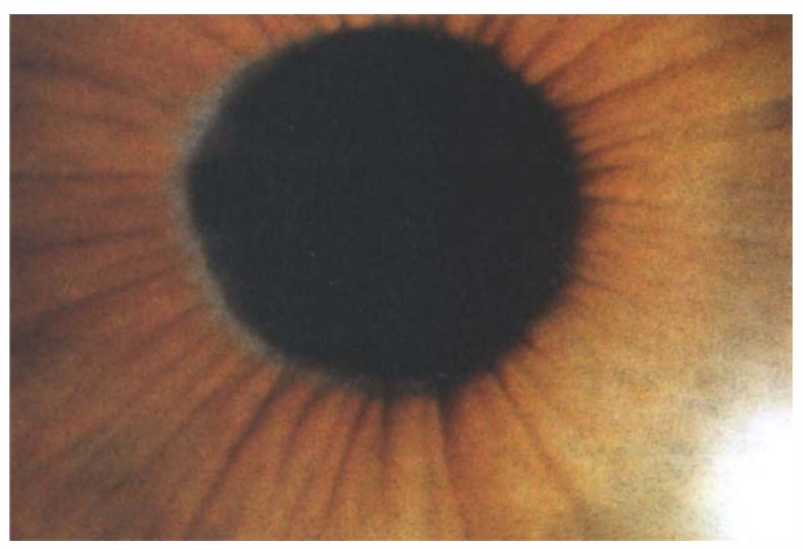

Fig. 1. Right eye: aphakic, pseudoexfoliative material (PXM) at the pupillary margin. 


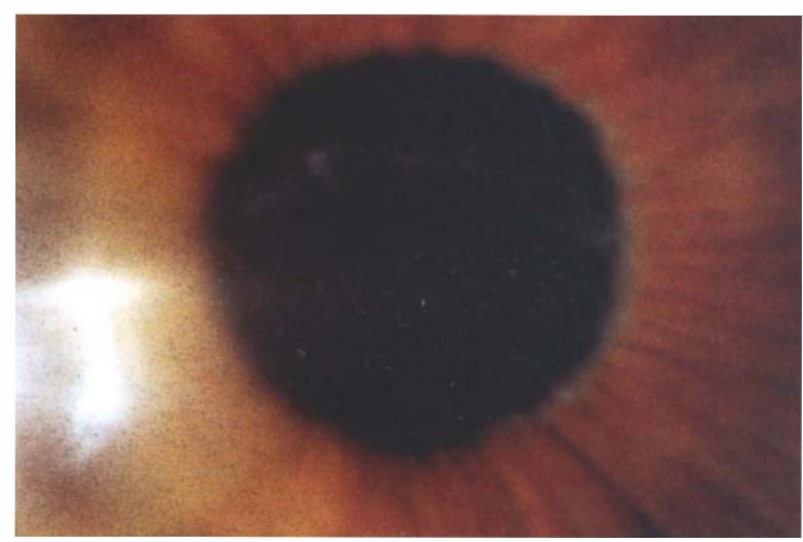

Fig. 2. Left eye: subluxated lens, PXM at superior equator of lens, PXM at pupillary margin.

\section{References}

1. Prince AM, Ritch R. Clinical signs of the pseudoexfoliation syndrome. Ophthalmology 1986;93:803-7.

2. Carpel EF. Pupillary dilatation in eyes with pseudo-exfoliation syndrome. Am J Ophthalmol 1988;105:692-4.

3. Schlotzer-Schrehardt U, Naumann GOH. A histopathologic study of zonular instability in pseudoexfoliation syndrome. Am J Ophthalmol 1994;118:730-43.

4. Streeten BW, Li ZY, Wallace RN, et al. Pseudoexfoliative fibrillopathy in visceral organs of a patient with pseudoexfoliation syndrome. Arch Ophthalmol 1992;110:1757-62.
5. Streeten BW, Bookman L, Ritch R, et al. Pseudoexfoliative fibrillopathy in the conjunctiva: a relation to elastic fibers and elastosis. Ophthalmology 1987;94:1439-49.

6. Mitchell P, Wang JJ, Smith W. Association of pseudoexfoliation syndrome with increased vascular risk. Am J Ophthalmol 1997;124:685-7.

7. Freissler K, Kuchle M, Naumann GOH. Spontaneous dislocation of the lens in pseudoexfoliation syndrome. Arch Ophthalmol 1995;113:1095-6.

Ajai K. Tyagi

Peter J. McDonnell

Birmingham \& Midland Eye Centre

Dudley Road

Birmingham B18 7QU, UK

Mr A.K. Tyagi, MS, FRCS

Department of Ophthalmology

Claremont Wing

Royal Victoria Infirmary

Queen Victoria Road

Newcastle upon Tyne NE1 4LP, UK

Tel: +44 (0)1912325131

Fax: +44 (0)1912275246 